\title{
Die Zulassungsvorlage für Leistungserbringer vor dem Finale
}

\section{Bruno Henggi}

Verantwortlicher Public Affairs der FMH

\author{
Seit zwei Jahrzehnten versucht das Parlament, eine dauerhafte gesetzliche Grund- \\ lage für die Zulassung von Ärztinnen und Ärzten auszuarbeiten. Seit 2018 beraten \\ die Räte eine definitive und umfassende Vorlage zur Zulassungssteuerung. Ob sie \\ vom neuen Parlament im Frühjahr nächsten Jahres verabschiedet oder das Schick- \\ sal früherer Versuche teilen wird, ist offen.
}

Die Ursprünge der aktuellen Diskussion über die Zulassung von Leistungserbringern und den politischen Willen, die Zahl der frei praktizierenden ambulant tätigen Ärztinnen und Ärzte zu steuern und zu kontrollieren, reichen in die Zeit unmittelbar nach Inkraftsetzung des KVG und dem Abschluss der Bilateralen Verträge I zurück.

Auf den 1. Januar 2001 wurde erstmals die Kompetenz des Bundesrats festgelegt, eine auf drei Jahre beschränkte Bedürfnisklausel für die Zulassung einzuführen. Dafür ausschlaggebend waren die Kostenentwicklung im ambulanten Bereich in den 90er Jahren zusammen mit Befürchtungen, dass durch das Freizügigkeitsabkommen mit der damaligen EU-15, das 2002 in Kraft trat, noch zusätzliche Kostendynamik entstehen würde.

Dem Bundesrat wurde die Kompetenz erteilt, die Zulassung der ambulant tätigen Ärztinnen und Ärzte von einem Bedürfnis abhängig zu machen. Von dieser Regelung betroffen sind die selbständig oder unselbständig arbeitenden Ärztinnen und Ärzte, die ihre Tätigkeit in Praxen oder im ambulanten Bereich von Spitälern ausüben. Seit 2013 bestimmt eine Ausnahmeregelung, dass für Personen kein Bedürfnisnachweis erforderlich ist, welche mindestens drei Jahre an einer anerkannten schweizerischen Weiterbildungsstätte gearbeitet haben. Darüber hinaus können die Kantone die Zulassung der Ärzte an Bedingungen knüpfen.

\section{Lange Vorgeschichte}

Seine Kompetenz zur Zulassungssteuerung nahm der Bundesrat mit der ersten Verordnung vom 3. Juli 2002 wahr. Die Zulassungsbeschränkung wurde in der Folge dreimal verlängert und lief Ende 2011 aus. Wegen der starken Zunahme freipraktizierender Ärztinnen und Ärzte in gewissen Regionen wurde sie Mitte 2013 mit einem dringlichen Bundesbeschluss wieder für drei Jahre eingeführt. Im Jahr 2015 scheiterte eine Gesetzvorlage mit einer Stimme Differenz in der Schlussabstimmung, welche eine dauerhafte Lösung für die Zulassungssteuerung anstrebte, worauf ab 2016 erneut die auf drei Jahre befristete Bedürfnisklausel galt. Diese wurde zuletzt bis 2021 verlängert und soll nun von einer dauerhaften Zulassungssteuerung abgelöst werden.

\section{Die neue Gesetzvorlage soll die Zulassung umfassender regulieren als bisher.}

Die neue Gesetzvorlage, welche seit 2018 im Parlament beraten wird, soll nicht bloss die befristete Regelung ablösen, sondern die Zulassung weit umfassender regulieren als bisher. Erstens sollen neu die Qualität und Wirtschaftlichkeit der Leistungen durch eine Erhöhung der Anforderungen an die Leistungserbringer gesteigert werden. Dem Bundesrat wird die Kompetenz gegeben, Auflagen namentlich im Bereich der Qualitätsentwicklung festzulegen und sie mit dem Zulassungsentscheid zu verbinden. Zweitens wird ein formales Zulassungsverfahren eingeführt. Und drittens geben die neuen Bestimmungen den Kantonen die Möglichkeit, das Versorgungsangebot selber nach ihrem Bedarf zu regulieren.

\section{Qualitative Verbesserung}

Das Parlament hat richtigerweise die Bestimmungen des formalen Zulassungsverfahrens in der vom Bundesrat vorgeschlagenen Fassung stark korrigiert, bei 
der geltenden Regelung angesetzt und sie weiter verschärft. Denn sie gilt als Erfolg: «Die [...] Weiterführung der Einschränkung der Zulassung zur Tätigkeit zulasten der obligatorischen Krankenpflegeversicherung bietet den Kantonen, die darauf angewiesen sind, ein wirksames Steuerungsinstrument [...] Es geht nicht um einen Zulassungsstopp, sondern es geht um eine begrenzte Zulassungssteuerung für Ärzte, welche sich nicht über eine dreijährige Weiterbildung an einer schweizerisch anerkannten Weiterbildungsstätte ausweisen können. Kantone, welche keinen Handlungsbedarf haben, müssen nichts tun. Die Vorlage ist föde-

\section{Die Ärzteschaft hatte sich mit zwei Qualitäts- kriterien in die Diskussion eingebracht.}

ralistisch, und die situationsgerechte Interventionsmöglichkeit dient der Qualitätssicherung und der Patientensicherheit.» Mit diesen Aussagen warb Nationalrätin Ruth Humbel 2016 für eine weitere Verlängerung der befristeten Zulassungssteuerung. ${ }^{1}$ Die Ärzteschaft hatte sich mit zwei Qualitätskriterien in die Diskussion eingebracht und schlug erstens vor, das bestehende Dreijahreskriterium zu verschärfen: Anstatt «Wer drei Jahre an einer anerkannten schweizerischen Weiterbildungsstätte gearbeitet hat, erhält die Zulassung» soll neu die dreijährige Tätigkeit in der beantragten Fachdisziplin erfolgen. Die limitierte Verfügbarkeit von Weiterbildungsstätten für die fachspezifische Tätigkeit wird die Zulassungen für die Spezialdisziplinen begrenzen. Das zweite neu ins KVG aufgenommene Qualitätskriterium betrifft das Spracherfordernis. Ärztinnen und Ärzte müssen die in ihrer Tätigkeitsregion erforderliche Sprachkompetenz in einer in der Schweiz abgelegten Sprachprüfung nachweisen. Die Nachweispflicht muss vor Antritt der ärztlichen Berufstätigkeit erbracht werden. Sie soll aber für Ärztinnen und Ärzte entfallen, die über eine schweizerische gymnasiale Maturität verfügen. Mit den verschärften Sprachanforderungen B2 kann im Berufsalltag sichergestellt werden, dass sich der Arzt im Team mit Gesundheitsfachpersonen und Arbeitskollegen und mit Patientinnen und Patienten differenziert und fliessend verständigen kann.

Sondersession des Nationalrats vom April 2016; vierte Sitzung vom 27.4.2016, um 8 Uhr; 16.401.

bruno.henggi[at]fmh.ch tens des Berichterstatters der Gesundheitskommission des Rates abgegeben. So wurde ausgeführt, dass beide Maturitäten für die Zulassung genügen und beide Maturitäten von der Nachweispflicht der Sprachkompetenz entbinden. Und wenn die Sprache in der Tätigkeitsregion Gegenstand der bestehenden Maturitätsprüfung ist, wird von einer Prüfung abgesehen. Darüber hinaus wurde im Namen der Mehrheit der ständerätlichen Gesundheitskommission erklärt, dass ein Zürcher Arzt, der in Genf tätig sein will, keine Sprachprüfung ablegen müsse. Eine kantonale schweizerische Maturität, die auf Deutsch abgeschlossen werde, beinhalte in genügendem Niveau Französisch. Und rein formell sollte eine abgeschlossene schweizerische Maturität genügen. Das hiesse, ein Arzt mit Schweizer Matur sollte keine Sprachprüfung ablegen müssen: dies der Wille der Gesundheitskommission des Ständerats.

Einen Schritt in Richtung Kompromiss unternahm der Ständerat auch bei den Höchstzahlen, deren Festsetzung im Kompetenzbereich der Kantone sein wird. Es wäre ausreichend gewesen, wenn der Ständerat ent-

\section{Ein Arzt mit Schweizer Matur sollte keine Sprachprüfung ablegen müssen.}

schieden hätte, den Kantonen die Möglichkeit einzuräumen, für ambulant tätige Ärztinnen und Ärzte Höchstzahlen einzuführen. Stattdessen wählte er die Option Überregulierung und votierte für eine verpflichtende Bestimmung. Allerdings ist dem Ständerat zugutezuhalten, dass er sich dafür ausgesprochen hat, die Kantone nicht zu verpflichten, die Zulassung von Leistungserbringern zu stoppen, wenn die Kosten in einem Fachgebiet überdurchschnittlich steigen. Die vom Ständerat beschlossene Anwendung von Höchstzahlen wäre deshalb insgesamt annehmbar, sofern der Beschluss der kleinen Kammer Bestand hält und die Versicherer das Beschwerderecht gegen kantonale Entscheide über Höchstzahlen effektiv nicht erhalten. Bereits zu einem früheren Zeitpunkt war die Lockerung des Vertragszwangs auf Druck des Ständerats aus der Zulassungsvorlage gekippt worden. In der dritten Lesung und der anschliessenden Einigungskonferenz die Beratung wird in der Frühjahrssession fortgesetzt wird es im Wesentlichen darum gehen, die grosse Differenz wegen der Verknüpfung der Zulassungsvorlage mit dem Geschäft «einheitliche Finanzierung der Leistungen im ambulanten und stationären Bereich» zu eliminieren. An dieser Differenz könnte der erneute Versuch des Gesetzgebers, die Zulassung von Ärztinnen und Ärzten dauerhaft zu regeln, erneut scheitern. 